\title{
Factors Affecting Short-Term Outcome of Critically III Children with Malignancies Admitted in Pediatric Intensive Care Unit: A Retrospective Observational Study
}

\author{
Priyanka Aggarwal ${ }^{1} \quad$ Ishan Kumar ${ }^{2} \quad$ Sunil Kumar Rao Pradhap K $^{3} \quad$ Vineeta Gupta \\ ${ }^{1}$ Department of Pediatrics, Division of Pediatric Hematology \\ Oncology, Institute of Medical Sciences, Banaras Hindu University, \\ Varanasi, Uttar Pradesh, India \\ 2Department of Radiodiagnosis, Institute of Medical Sciences, \\ Banaras Hindu University, Varanasi, Uttar Pradesh, India

\begin{abstract}
Address for correspondence Priyanka Aggarwal, MBBS, MD (Pediatrics) Department of Pediatrics, Division of Pediatric Hematology Oncology, Institute of Medical Sciences, Banaras Hindu University, Varanasi 221005, Uttar Pradesh, India
\end{abstract} \\ (e-mail: tamanna.horizon@gmail.com).
}

${ }^{3}$ Department of Pediatrics, Division of Pediatric Intensive Care and Pulmonology, Institute of Medical Sciences, Banaras Hindu University, Varanasi, Uttar Pradesh, India

Ind J Med Paediatr Oncol 2021;42:333-338.

\begin{abstract}
Keywords

- pediatric cancer

- outcome

- PICU

Introduction Pediatric cancer contributes $<1 \%$ of all malignancies. Childhood cancer survival has improved dramatically with the use of more intensive chemotherapy regimens, better stratification, and improvement in supportive care with enhanced facilities in pediatric intensive care unit (PICU).

Objective The aim of this study was to identify the risk factors responsible for poor outcome in critically ill children with malignancies admitted in PICU.

Materials and Methods Sixty-four children with a primary diagnosis of malignancy admitted in PICU with disease or treatment related complications were enrolled retrospectively. The short-term outcome, that is, shifting from PICU to ward, was assessed in relation to the presence of febrile neutropenia, organ failure, hepatitis, acute renal failure as well as requirement of inotropes and mechanical ventilation. Death was considered as an adverse outcome in this study.

Results The mean age of study population was $6.25 \pm 3.91$ and M:F ratio 2.4:1. The majority of children had hematological malignancies (81.25\%), that is, pre-B acute lymphoblastic leukemia (ALL) (45.3\%), non-Hodgkin lymphoma (21.3\%), acute myeloid leukemia (12.5\%), T ALL (10.9\%), and Hodgkin lymphoma (3.1\%). Few children also had retinoblastoma (4.7\%) and Langerhans cell histiocytosis (1.6\%). The mean duration of PICU stay was $3.16 \pm 2.31$ days. Sepsis (37.5\%) was the most common indication for PICU admission, followed by metabolic disturbance (26.6\%), respiratory failure (17.2\%), neurological complaints (15.6\%), and anaphylactic shock (3.1\%). Children requiring mechanical ventilation $(p<0.001)$, inotrope support $(p<0.001)$, having acute renal failure $(p=0.001)$, and $>1$ organ failure $(p<0.001)$ were associated with adverse outcome. The overall survival at the time of discharge from PICU was $64 \%$.

Conclusion In the context of low- and middle-income countries, optimal resource utilization by early identification of risk factors for clinical deterioration is required to allow timely admission to PICU and delivery of life-saving therapy to salvageable patients.
\end{abstract}

DOI https://doi.org/ 10.1055/s-0041-1733824 ISSN 0971-5851
(C) 2021. Indian Society of Medical and Paediatric Oncology. This is an open access article published by Thieme under the terms of the Creative Commons Attribution-NonDerivative-NonCommercial-License, permitting copying and reproduction so long as the original work is given appropriate credit. Contents may not be used for commercial purposes, or adapted, remixed, transformed or built upon. (https://creativecommons.org/licenses/by-nc-nd/4.0/). Thieme Medical and Scientific Publishers Private Ltd. A-12, Second Floor, Sector -2, NOIDA -201301, India 


\section{Introduction}

Pediatric cancer contributes $<1 \%$ of all malignancies. ${ }^{1}$ The survival of children with cancer has improved dramatically over the past few decades with 5-year survival rates approaching to $\sim 80 \%$ from $40 \%$ in developed countries ${ }^{1,2}$ due to use of more intensive chemotherapy regimens, better stratification, and substantial advances in supportive care. Unfortunately, survival data from low- and middle-income countries is scarce. ${ }^{2}$

Pediatric intensive care unit (PICU) is an important aspect of supportive care. Children with cancer are admitted to PICU for varied reasons, that is, tumor-related problems like superior vena cava (SVC) syndrome and tumor lysis syndrome (TLS), or therapy-related toxicity and/or immunosuppression resulting into infectious complications., ${ }^{3,4}$ Most of the data regarding PICU outcomes is available from developed countries. There are studies that look at outcome of children admitted in PICU where adverse outcome was reported if children required inotrope support or ventilation in context of sepsis after bone marrow transplantation., ${ }^{5,6}$ Few other studies conducted to identify prognostic factors at the time of PICU admission stressed on age (varied cut off levels), type as well as stage of malignancy, remission status, and response to chemotherapy. However, results of these studies were not uniform among other reports. ${ }^{7,8}$

In developing countries, as most centers lack PICU facilities for critically ill children with malignancy, similar data regarding PICU outcomes is limited. However, there are few studies to suggest requirement of mechanical ventilation, inotrope usage, and multiorgan dysfunction syndrome following sepsis as predictors of adverse outcome. ${ }^{9,10}$ Due to limited infrastructure, there is a need for identifying children in whom survival chances may improve if PICU facility is provided to them. Therefore, to improve survival in these children, it is prudent to identify risk factors responsible for poor outcome in critically ill children with malignancies admitted in PICU and thus the study was planned accordingly.

\section{Materials and Methods}

A retrospective observational study including children with a primary diagnosis of malignancy who were admitted from 1 December 2015 to 30 October 2019 in the PICU at a tertiary hospital from North India was planned.

Inclusion criteria:

1. Children with hematological or nonhematological malignancy who qualified PICU admission criteria and got admitted in PICU.

\section{Exclusion criteria:}

1. Children with hematological or nonhematological malignancy who required PICU admission but could not be admitted due to nonavailability of beds in PICU.

2. Data incomplete or missing.

Data was collected from hospital records including age, sex, initial diagnosis, indication of admission, comorbidities, need of vasopressors, requirement for ventilation, presence of organ failure, duration of stay, and the short-term outcome.

\section{Criteria for PICU Admission}

I. All newly diagnosed children with malignancy (hematological or nonhematological) were admitted, if they had tumor-related complications, that is,

1. Life-threatening respiratory compromise, that is, high supplemental $\mathrm{O}_{2}$ requirement (fraction of inspired oxygen $\geq 0.5$ ) and/or potential need for emergency endotracheal intubation and mechanical ventilation, as a consequence to

i. SVC syndrome.

ii. Pulmonary/pleural metastasis.

iii. Peritoneal metastasis/large intraabdominal tumor.
2. Clinical/laboratory TLS $^{11}$ (as per Cairo Bishop Classification).
3. Circulatory failure.

II. Children receiving chemotherapy were admitted, as a consequence of therapy-related complications, that is,

1. Anaphylactic shock.

2. Severe sepsis with/without respiratory and/or hemodynamic instability.

3. Neurological complaints, that is, status epilepticus and neurological weakness (if there was associated respiratory compromise/autonomic instability).

4. Metabolic disturbance (hypoglycemia, hypo/hypercalcemia, hypo/hyperkalemia, hypernatremia).

5. Progressive disease with respiratory and/or circulatory failure.

Blood and urine cultures were sent for all patients admitted with a baseline diagnosis of neutropenic sepsis. Those children who developed neutropenic sepsis during PICU stay were subjected to cultures from blood, urine, and/or bronchoalveolar lavage as per PICU protocol. Sepsis and organ dysfunction was diagnosed according to International Pediatric Sepsis Consensus Conference: definition for sepsis and organ dysfunction in pediatrics. ${ }^{12}$

Children were admitted to PICU from outpatient department or ward depending on the availability of beds in PICU. Outcome from PICU was assessed as discharge from PICU or death of the child.

\section{Statistical Analysis}

SPSS (SPSS Inc., Chicago, Illinois, United States) for windows was used for data entry and analysis. All numerical variables are expressed as median with range. For comparison of categorical data, chi-squared test was used. For categorical variables with cell values $<5$, Fisher's exact test was used. A $p$-value $<0.05$ was taken as significant. Receiver operating characteristic (ROC) curve was plotted for number of inotropes to predict mortality and cutoff value was derived using coordinates of the curve. Binary logistic regression analysis 
with hierarchical entry of categorical variables were used to predict mortality in our cohort.

\section{Ethics}

The procedures followed were in accordance with the ethical standards of the responsible committee on human experimentation (institutional) and with the Helsinki Declaration of 1964, as revised in 2013. Ethical clearance from Institute Ethical Committee of Institute of Medical Sciences, Banaras Hindu University, was obtained (dated 24.09.2020, No Dean/2020/EC/2132). A waiver of informed consent was obtained from the Ethics Committee.

\section{Results}

Sixty-four children (2.5\%) were admitted to PICU with a primary diagnosis of malignancy. The mean age of these children was $6.25 \pm 3.91$ years and male:female ratio was $2.4: 1$. Hematological malignancies accounted for $81.25 \%$ of the total admissions, that is, pre-B acute lymphoblastic leukemia

Table 1 Characteristics of the study population

\begin{tabular}{|c|c|}
\hline Characteristic & Result \\
\hline \multicolumn{2}{|l|}{ Age $(y)$} \\
\hline Mean \pm SD & $6.25 \pm 3.91$ \\
\hline \multicolumn{2}{|l|}{ Gender } \\
\hline Male, $n(\%)$ & $45(70.3)$ \\
\hline Female, $n(\%)$ & $19(29.7)$ \\
\hline \multicolumn{2}{|l|}{ Diagnosis } \\
\hline Pre-B ALL, $n(\%)$ & $29(45.3)$ \\
\hline $\mathrm{NHL}, n(\%)$ & $14(21.9)$ \\
\hline AML, $n(\%)$ & $8(12.5)$ \\
\hline T ALL, $n(\%)$ & $7(10.9)$ \\
\hline $\mathrm{RB}, n(\%)$ & $3(4.7)$ \\
\hline $\mathrm{HL}, n(\%)$ & $2(3.1)$ \\
\hline $\mathrm{LCH}, n(\%)$ & $1(1.6)$ \\
\hline \multicolumn{2}{|l|}{ Indication for PICU admission } \\
\hline Sepsis, $n(\%)$ & $24(37.5)$ \\
\hline Metabolic disturbances, $n$ (\%) & $17(26.6)$ \\
\hline Respiratory failure, $n(\%)$ & $11(17.2)$ \\
\hline Neurological complaints, $n$ (\%) & $10(15.6)$ \\
\hline Anaphylactic shock, $n(\%)$ & $2(3.1)$ \\
\hline \multicolumn{2}{|l|}{ Duration of stay (d) } \\
\hline Median & 3 \\
\hline Range & $1-13$ \\
\hline Mechanical ventilation, $n(\%)$ & $24(37.5)$ \\
\hline \multicolumn{2}{|l|}{ Days of ventilation (d) } \\
\hline Median & 1 \\
\hline Range & $1-6$ \\
\hline
\end{tabular}

Abbreviations: ALL, acute lymphoblastic leukemia; AML, acute myeloid leukemia; LCH, Langerhans cell histiocytosis; NHL, non-Hodgkin lymphoma; PICU, pediatric intensive care unit; RB, retinoblastoma; SD, standard deviation.
(ALL) $(29 ; 45.3 \%)$, non-Hodgkin lymphoma (14; 21.3\%), acute myeloid leukemia (8; 12.5\%), T ALL (7; 10.9\%), and Hodgkin lymphoma $(2 ; 3.1 \%)$. Few children had retinoblastoma (3; 4.7\%) and Langerhans cell histiocytosis (1; 1.6\%).

\section{Sepsis}

Sepsis $(24 ; 37.5 \%)$ was the most common indication for admission, followed by metabolic disturbance $(17 ; 26.6 \%)$, respiratory failure $(11 ; 17.2 \%)$, neurological complaints (10; 15.6\%), and anaphylactic shock $(2 ; 3.1 \%)$ (-Table 1). Children admitted for sepsis included those with septic shock (8; 33.3\%), pneumonia (not requiring oxygen supplementation) $(7 ; 29 \%)$, neutropenic enterocolitis $(7 ; 29 \%)$, severe malaria $(1 ; 4 \%)$, and severe dengue $(1 ; 4 \%)$.

As per PICU protocol, 102 specimens including cultures from blood, urine, pleural fluid, bronchoalveolar fluid, and/or stool of children admitted with a baseline diagnosis of neutropenic sepsis $(n=24)$ or those who developed neutropenic sepsis during PICU stay ( $n=14$ ) were sent. Fifteen organisms were isolated from 102 specimens. Among these, nine (60\%) isolates were Gram-negative organisms, that is, Klebsiella pneumonia (3; 20\%) followed by Escherichia coli (2; 13.3\%), Acinetobacter baumannii (2; 13.3\%), and Citrobacter spp. (2; 13.3\%). Staphylococcus aureus was reported as a causative organism in four (26.6\%) cases and Candida spp. in two (13.3\%) cases. Five organisms were isolated from cultures that were sent from children who developed neutropenic sepsis during hospital stay. Majority of the isolates were Gram-negative, that is., Acinetobacter baumannii $(n=2)$ and Citrobacter sp. ( $n$ $=1$ ) followed by Candida spp. $(n=2)$. In our patient cohort, febrile neutropenia was recognized as an independent risk factor for mortality $(p=0.003)$.

\section{Metabolic Disturbances}

Another important cause of PICU admission in our cohort was electrolyte abnormalities. This was encountered as a component of TLS or in isolation. Twenty-six children experienced either hypocalcemia (8; 12.5\%), hypokalemia (7; 10.9\%), both hypocalcemia and hypokalemia (6;9.4\%), or hypercalcemia $(5 ; 7.8 \%)$.

\section{Respiratory Failure}

Children were also admitted for respiratory failure. This group comprised of children who presented with respiratory distress due to presence of SVC syndrome, disease-related pulmonary metastasis, pleural effusion and/or ascites, and pneumonia required oxygen supplementation.

\section{Neurological Complaints}

Among those admitted for neurological complaints, status epilepticus was the most common indication. In these children, the cause of seizures was posterior reversible encephalopathy during induction chemotherapy $(n=4)$, infective meningitis $(n=2)$, intracranial bleed $(n=2)$, and malignant cell infiltration in the central nervous system owing to progressive disease $(n=1)$. One child was admitted as he had Guillain-Barré syndrome with respiratory involvement. 
Table 2 Prognostic factors affecting outcome of children admitted in pediatric intensive care unit

\begin{tabular}{|l|l|l|l|}
\hline & Discharged, $\boldsymbol{n}$ & Expired, $\boldsymbol{n}$-Value & 0.069 \\
\hline Age & $5.59 \pm 3.54$ & $7.43 \pm 4.31$ & \\
\hline Gender & & & 0.160 \\
\hline Male, $n(\%)$ & $32(71)$ & $13(29)$ & \\
\hline Female, $n(\%)$ & $9(47)$ & $10(53)$ & 0.003 \\
\hline Febrile neutropenia & 11 & 15 & $<0.001$ \\
\hline$>1$ organ failure & 1 & 4 & 0.215 \\
\hline Hepatitis & 3 & 7 & 0.001 \\
\hline Acute renal failure & 1 & 21 & $<0.001$ \\
\hline Inotrope support & 10 & 19 & $<0.001$ \\
\hline Mechanical Ventilation & 5 & & \\
\hline
\end{tabular}

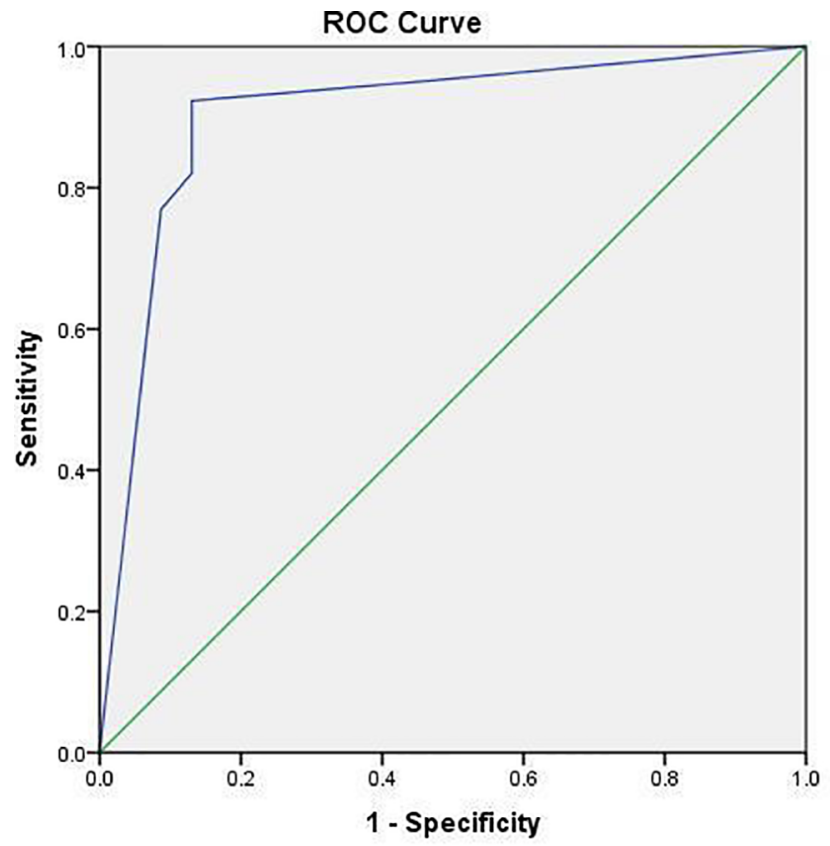

Diagonal segments are produced by ties.

Fig. 1 Receiver operating characteristic (ROC) curve to predict mortality for number of inotropes used.

The median duration for PICU stay was 3 days (1-13 days). Twenty-four children required mechanical ventilation for a median duration of 1 day (1-6 days).

The overall survival at the time of discharge from PICU was $64 \%$ and did not correlate with age and gender. Organ failure occurred on 35 occasions. Circulatory failure was the most prominent (31 occasions) followed by respiratory failure (24 occasions), renal failure (8 occasions), and hepatic failure (7 occasions). Thirteen children developed more than one organ failure that was associated with statistically significant adverse outcome (i.e., $p<0.001$ ). Requirement of mechanical ventilation due to respiratory failure $(p<0.001)$, presence of acute kidney injury $(p=0.001)$, and circulatory failure as evidenced by use of inotropes $(p<0.001)$ was associated with poor outcome. Seven children also developed acute hepatitis, but it was not associated with poor outcome $(p=0.215 ;$ - Table 2).

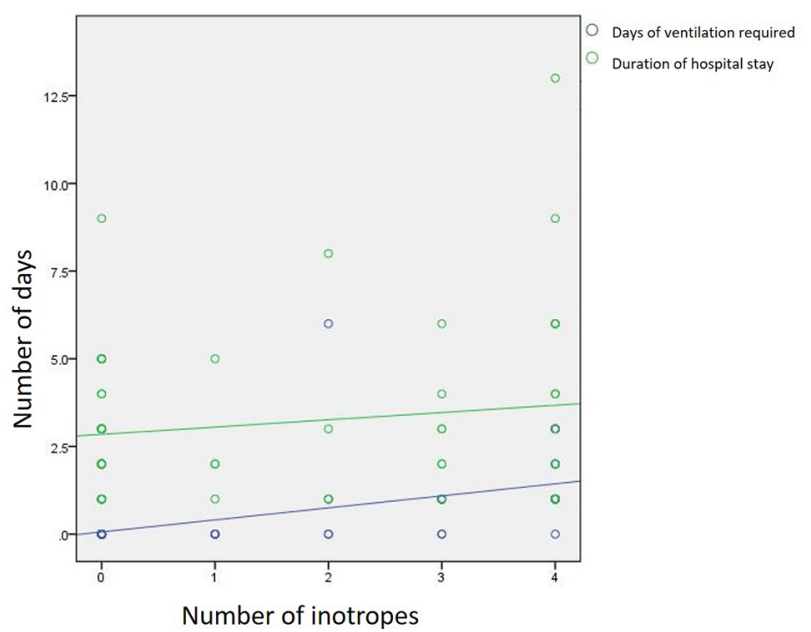

Fig. 2 Scatter plot between the number of inotropes (X-axis) and duration of mechanical ventilation (purple) as well as the duration of pediatric intensive care unit stay (green) on Y-axis showing linear relation.

ROC curve was plotted to predict mortality for number of inotropes used that revealed an area under curve 0.905 . Coordinates of the curve yielded a cutoff value of more than 2 for number of inotropes predicting mortality with $92.3 \%$ sensitivity and $87 \%$ specificity (-Fig. 1). A scatter plot suggested that number of inotropes showed linear relation with the duration of mechanical ventilation as well as the duration of PICU stay ( - Fig. 2). Binary logistic regression model (-Table 3) could explain $73.6 \%$ mortality. History of more than two inotrope administrations was the only significant predictor of mortality.

\section{Discussion}

Children with malignancy contribute $<10 \%$ of total PICU admissions worldwide. ${ }^{13,14}$ They remain the most complex and challenging group with significantly worse outcome as compared with children admitted to PICU for other illnesses. ${ }^{15,16}$ The mortality of pediatric cancer patients admitted to PICU worldwide is high $(28 \%)^{17}$ as it was in this study (36\%). This is partly because these children become 
Table 3 Summary of logistic analysis for variables predicting mortality in our patients

\begin{tabular}{|l|l|l|l|l|}
\hline Predictor & Odds ratio & SE & 95\% Cl & $p$-Value \\
\hline Febrile neutropenia & 0.942 & 1.19 & $0.091-9.79$ & 0.96 \\
\hline$>1$ organ failure & 6.19 & 1.58 & $0.27-137.9$ & 0.249 \\
\hline Acute renal failure & 2.79 & 2.02 & $0.05-148.3$ & 0.61 \\
\hline Inotrope support (>2 inotropes) & 19.5 & 1.19 & $1.4-260.8$ & 0.025 \\
\hline Mechanical ventilation & 4.0 & 1.0 & $0.48-33.8$ & 0.195 \\
\hline
\end{tabular}

Abbreviations: $\mathrm{Cl}$, confidence interval; SE, standard error.

immunocompromised during treatment and develop life-threatening infectious complications that need aggressive antibiotic policy. Also, children presenting with advanced malignancy with various malignancy-related complications require intensive care along with aggressive blood product support.

In accordance with previous studies, sepsis (37.5\%) and respiratory failure $(17.2 \%)$ were the most common indications for PICU admissions..$^{6,18,19}$ However, in the present study metabolic disturbances also contributed to $26.6 \%$ of the PICU admissions. Metabolic disturbance as an indication for PICU admission has not been considered separately in previous studies. . $^{9,19,20}$

Although boys were admitted more as compared with girls, mortality was higher among girls (i.e., 53 vs. 29\%) with no statistical significance. This may be because girls presented with very severe symptoms as compared with boys at the time of diagnosis. Ali et al. in a similar study conducted in Egyptian children found the cause of male preponderance is poor socioeconomic status and special preference of parents for male children in seeking treatment. ${ }^{21}$

The majority of children experiencing metabolic disturbances were those who developed hypocalcemia due to TLS or developed hypocalcemia and/or hypokalemia due to coexistence of malnutrition during intensive chemotherapy. Although the presence of metabolic disturbance was an important indication for PICU admission, it was not associated with statistically significant adverse outcome. However, as metabolic disturbances can be corrected with appropriate and timely intervention, we want to emphasize their early identification and appropriate management for improving outcome.

Children in this study had a survival rate of $64 \%$, which is comparable with previous reports. In retrospective studies by van Veen et al,22 among 51 patients over 10 years, and Heney et al, ${ }^{18}$ among 70 patients over 6 years, a survival rate of 70 and $51 \%$ has been reported, respectively.

The single most important predictor of death in our study was multiorgan failure. Among 13 children who developed multiorgan failure, 12 children died in this study. As with general population, mortality exceeding $70 \%$ has been reported by various researchers if $\geq 3$ organs are involved. ${ }^{19,23,24}$ Most of the children who developed acute renal failure during PICU stay had a statistically significant adverse outcome that is in accordance with studies performed in children ${ }^{25}$ and adults ${ }^{26}$ with cancer. Although mortality was more if the child developed hepatitis in isolation, it was not significant statistically.

Also, as observed across other studies, 9,10,19,21,24 cohort of children requiring inotropic support and mechanical ventilation had a high mortality, that is, 79 and $64 \%$ respectively. Thus, presence of multiorgan dysfunction, acute renal failure, requirement of multiple inotropes, and mechanical ventilation can be taken as predictors of adverse outcome. However, use of more than 2 inotropes was recognized as the most important predictor of adverse outcome among all these parameters.

The limitations in our study were small sample size, nonquantification of various metabolic disturbances, nonavailability of data regarding patients who were denied admission to PICU due to nonavailability of beds, as it was a retrospective analysis.

\section{Conclusion}

PICU outcomes and resource utilization have not been studied rigorously. Most of the information available is by means of single-center retrospective studies. Thus, there is a need of large multicentric prospective interventional studies that compare PICU outcomes for pediatric cancer population, which would improve understanding about underlying mechanisms for organ dysfunction, early identification of risk factors for clinical deterioration, and prioritization of patients that are salvageable, thereby allowing timely admission to PICU and delivery of life-saving therapy.

\section{Source of Funding}

None

\section{Conflict of Interest}

None

\section{References}

1 Steliarova-Foucher E, Stiller C, Kaatsch P, et al. Geographical patterns and time trends of cancer incidence and survival among children and adolescents in Europe since the 1970s (the ACCISproject): an epidemiological study. Lancet 2004;364(9451):2097-2105

2 Bhakta N, Force LM, Allemani C, et al. Childhood cancer burden: a review of global estimates. Lancet Oncol 2019;20(1):e42-e53

3 Piastra M, Fognani G, Franceschi A; ICARO Italian Network For Intensive Care In Pediatric Oncology. Pediatric intensive care unit admission criteria for haemato-oncological patients: 
a basis for clinical guidelines implementation. Pediatr Rep 2011;3(2):e13

4 Haase R, Mathony U, Lieser U, Nagel F, Sitka U, Burdach S. [Oncology patients in a pediatric intensive care unit-a 7-year experience]. Klin Padiatr 2003;215(4):234-240

5 Heying R, Schneider DT, Körholz D, Stannigel H, Lemburg P, Göbel U. Efficacy and outcome of intensive care in pediatric oncologic patients. Crit Care Med 2001;29(12):2276-2280

6 Hallahan AR, Shaw PJ, Rowell G, O'Connell A, Schell D, Gillis J. Improved outcomes of children with malignancy admitted to a pediatric intensive care unit. Crit Care Med 2000;28(11):3718-3721

7 Evison J, Rickenbacher P, Ritz R, et al. Intensive care unit admission in patients with haematological disease: incidence, outcome and prognostic factors. Swiss Med Wkly 2001;131(47-48):681-686

8 Hayes C, Lush RJ, Cornish JM, et al. The outcome of children requiring admission to an intensive care unit following bone marrow transplantation. Br J Haematol 1998;102(3):666-670

9 Akhtar N, Fadoo Z, Panju S, Haque A. Outcome and prognostic factors seen in pediatric oncology patients admitted in PICU of a developing country. Indian J Pediatr 2011;78(8):969-972

10 Ramzan R, Ramzan SP, Gupta D, Arora S, Sanjeev A. Pediatric intensive care unit: an essential service to improve survival of childhood cancer in developing world. Indian J Pediatr 2012;79(12):1669-1670

11 Cairo MS, Bishop M. Tumour lysis syndrome: new therapeutic strategies and classification. Br J Haematol 2004;127(1):3-11

12 Goldstein B, Giroir B, Randolph A; International Consensus Conference on Pediatric Sepsis. International pediatric sepsis consensus conference: definitions for sepsis and organ dysfunction in pediatrics. Pediatr Crit Care Med 2005;6(1):2-8

13 Gullberg N, Kalzén H, Luhr O, et al. Scandinavian Critical Care Trials Group. Immediate and 5-year cumulative outcome after paediatric intensive care in Sweden. Acta Anaesthesiol Scand 2008;52(8):1086-1095

14 Owens C, Mannion D, O'Marcaigh A, Waldron M, Butler K, O'Meara A. Indications for admission, treatment and improved outcome of paediatric haematology/oncology patients admitted to a tertiary paediatric ICU. Ir J Med Sci 2011;180(1):85-89

15 Dalton HJ, Slonim AD, Pollack MM. Multicenter outcome of pediatric oncology patients requiring intensive care. Pediatr Hematol Oncol 2003;20(8):643-649
16 Tamburro RF, Barfield RC, Shaffer ML, et al. Changes in outcomes (1996-2004) for pediatric oncology and hematopoietic stem cell transplant patients requiring invasive mechanical ventilation. Pediatr Crit Care Med 2008;9(3):270-277

17 Wösten-van Asperen RM, van Gestel JP, van Grotel M, et al. POKER (PICU Oncology Kids in Europe Research group) research consortium. PICU mortality of children with cancer admitted to pediatric intensive care unit a systematic review and meta-analysis. Crit Rev Oncol Hematol 2019;142:153-163

18 Heney D, Lewis IJ, Lockwood L, Cohen AT, Bailey CC. The intensive care unit in paediatric oncology. Arch Dis Child 1992;67(3):294-298

19 Dursun O, Hazar V, Karasu GT, Uygun V, Tosun O, Yesilipek A. Prognostic factors in pediatric cancer patients admitted to the pediatric intensive care unit. J Pediatr Hematol Oncol 2009;31(7):481-484

20 Heba A. Sayed, Amany M. Ali, and Mahmoud M. Elzembely. Can Pediatric Risk of Mortality Score (PRISM III) Be Used Effectively in Initial Evaluation and Follow-up of Critically Ill Cancer Patients Admitted to Pediatric Oncology Intensive Care Unit (POICU)? A Prospective Study, in a Tertiary Cancer Center in Egypt. J Pediatr Hematol Oncol 2018;40(5):382-386

21 Ali AM, Sayed HA, Mohammed MM. The outcome of critically ill pediatric cancer patients admitted to the pediatric intensive care unit in a tertiary university oncology center in a developing country: a 5-year experience. J Pediatr Hematol Oncol 2016;38(5):355-359

22 van Veen A, Karstens A, van der Hoek AC, Tibboel D, Hählen $\mathrm{K}$, van der Voort $\mathrm{E}$. The prognosis of oncologic patients in the pediatric intensive care unit. Intensive Care Med 1996;22(3):237-241

23 Meyer S, Gottschling S, Biran T, et al. Assessing the risk of mortality in paediatric cancer patients admitted to the paediatric intensive care unit: a novel risk score? Eur J Pediatr 2005; 164(9):563-567

24 Ben Abraham R, Toren A, Ono N, et al. Predictors of outcome in the pediatric intensive care units of children with malignancies. J Pediatr Hematol Oncol 2002;24(1):23-26

25 Bion J. Cost containment: Europe. The United Kingdom. New Horiz 1994;2(3):341-344

26 Lanore JJ, Brunet F, Pochard F, et al. Hemodialysis for acute renal failure in patients with hematologic malignancies. Crit Care Med 1991;19(3):346-351 